\title{
NUESTRA EXPERIENCIA EN EL USO DE LA PRÓTESIS INTRAURETRAL UROLUME.
}

\author{
Cristobal García Peñalver, José Luis Parra Escobar y Eloy Sánchez Blasco.
}

Servicio de Urología. Hospital de Mérida. Mérida. Badajoz. España.

Resumen.- OBJETIVO: Urolume® es una endoprótesis uretral tipo stent, no magnética y autoexpandible indicada para el mantenimiento de la luz uretral en los casos de obstrucción infravesical. Ha sido aprobada por la Food and Drug Administration. Presentamos nuestra experiencia en el uso de esta prótesis en los casos de obstrucción por HBP y por estenosis uretral.

MÉTODOS: Desde abril de 1999 a julio de 2005 hemos colocado 18 endoprótesis Urolume en 17 varones; presentando 10 de ellos síntomas de HBP y 7 estenosis de uretra bulbar. Analizamos acontinuación los resultados de nuestra serie.

RESULTADOS: La edad media de los pacientes era de 61 años [30-79], siendo la media de edad de los pacientes con prótesis bulbar significativamente inferior. De los pacientes con HBP $(58,8 \%, n=10)$, el $80 \%(n=8)$ eran portadores de sonda lel $40 \%$ con riesgo quirúrgico
ASA III y el 60\% ASA IV). Han fallecido 4 pacientes, llevando sólo 1 sonda permanente en el momento del éxitus. La colocación de la endoprótesis se realizó con gel de xilocaína en 4 de estos 10 pacientes (40\%). La técnica ha fracasado en 2 pacientes, uno requiriendo de sonda permanente por retención urinaria lhasta que falleciól, y otro de extracción de la misma y adenomectomía por RAO. De entre los pacientes con estenosis de uretra $(41,2 \% n=7)$, en uno hemos tenido que colocar una segunda prótesis telescopada sobre la primera (por desplazamiento caudal de ésta), habiendo realizado además 2 RTU de tejido hiperplásico intraprotésico en el mismo paciente. Subjetivamente, la puntuación media en la escala de Madsen-Iversen precirugía era de 22,5 frente a 7,78 postcirugía $(p<0,005$; test de Wilconson). También hubo mejorías objetivas en las medias de los flujos máximos pre y postcirugía $15,7 \mathrm{ml} / \mathrm{s}$ a 20 , 9; $p<0,005$; test de Wilconson).

CONCLUSIONES: Se trata de una técnica segura y sencilla, pudiendo colocarse con anestesia local de forma ambulante. Presenta baja tasa de complicaciones, con mejoría significativa de los parámetros flujométricos y del cuestionario de sintomas. Constituyen una muy buena opción a tener en cuenta en pacientes ancianos, retencionistas crónicos y con alto riesgo quirúrgico. Pueden ser una alternativa válida a la cirugía en pacientes con estenosis bulbares cortas, en los que no se hayan realizado colgajos con piel y que no tengan espongiofibrosis profunda.

Palabras clave: Estenosis de uretra. Endoprótesis. HBP.

Summary.- OBJECTIVES: Urolume ${ }^{T M}$ is a stent type, non magnetic, self expanding urethral endoprosthesis indicated to keep the urethral lumen in cases of infravesical obstruction. It has been approved by the food and drug administration. We present our experience with 
the use of this prosthesis in cases of obstruction secondary to $\mathrm{BPH}$ and urethral stenosis.

METHODS: From April 1999 to July 2005 we implanted 18 Urolume ${ }^{T M}$ endoprosthesis in seventeen male patients; 10 of them had symptoms of BPH and 7 bulbar urethral stenosis. We analyze the results of our series.

RESULTS: Mean patient age was 61 years [30-79], being the mean age for patients with bulbar prosthesis significantly lower. Among patients with BPH 158.8\%, n $=10), 80 \%$ ( $n=8)$ had indwelling catheter $140 \%$ with ASA III surgical risk and 60\% ASA IV). 4 patients have died with only one of them having indwelling catheter at the time of death. The implantation of the endoprosthesis was performed with xylocaine gel in 4 of these 10 patients $(40 \%)$. The technique failed in two patients, one of them required indwelling catheter for urinary retention (to the time of his death), and the other one extraction of the prosthesis and prostatic adenomectomy for acute urinary retention. Among patients with urethral stenosis $(41.2 \%, n=7)$, we needed to implant a second prosthesis telescoped with the first one in one case /due to distal displacement), and to perform 2 transurethral resections of intraprosthesic hyperplastic tissue in the same patient. Subjectively, the mean value of the Madsen-Iversen score before surgery was 22.5 in comparison to 7.78 after surgery ( $p<0.005$; Wilcoxon test). There were also objectives improvements in mean maximal flow before and after surgery 15.7 to $20.9 \mathrm{ml} / \mathrm{sec}$.; $p<0.005$; Witcoxon test).

CONCLUSIONS: This is a safe and simple technique, which may be performed under local anesthesia as out patient surgery. It has a low complication rate, significantly improves the flowmetry parameters and symptom questionnaire results. It is a very good option to be taken into consideration in old patients, with chronic urinary retention, and high surgical risk. It may be a valid alternative to surgery in patients with short bulbar urethral stenosis, without previous skin flap urethroplasty and not having spongiofibrosis.

Keywords: Urethral stenosis. Urolume prosthesis. Lower urinary tract obstruction. BPH.

\section{INTRODUCCIÓN}

UroLume ${ }^{\circledR}$ es una endoprótesis uretral tipo stent, no magnética y autoexpandible indicada para el mantenimiento de la luz uretral en los casos de obstrucción infravesical. La idea de intentar mantener la luz uretral mediante un dispositivo auto-retentivo data de 1969 (1). Estos dispositivos han sido diseñados para permanecer de forma temporal o permanente. La principal diferencia entre estos dos tipos de dispositivos es que los primeros son intraluminales, $y$ se pueden asociar a incontinencia, estenosis de uretra, infección, incrustación o movilización . Los stents permanentes quedan recubiertos de urotelio siendo epitelizados en la mayoría de los casos evitando este tipo de problemas.

El stent es colocado bajo visión endoscópica directa, pudiendo repetirse ésta para confirmar la correcta ubicación del mismo, así como para diagnóstico y tratamiento de cualquier problema urológico concomitante.

Posiblemente, dentro de las alternativas terapeuticas disponibles para la obstrucción prostática, solamente los stents intraprostáticos alcanzan los mismos resultados que los obtenidos por cirugía prostática.

Presentamos nuestra experiencia en el uso de esta prótesis en los casos de obstrucción por HBP y por estenosis uretral.

\section{MATERIAL Y MÉTODOS}

Desde abril/1999 a julio/2005 hemos colocado 18 endoprótesis UroLume ${ }^{\circledR}$ en 17 varones presentando 10 de ellos síntomas de HBP y 7 de estenosis de uretra bulbar (Figura 1). Los criterios de exclusión y contraindicaciones quedan expuestos en la Tabla I.

Técnica de implante queda resumida en los siguientes pasos:

1) Profilaxis antibiótica según protocolo establecido con cefonicid o cefonicid y ceftriaxona si el paciente es portador de sonda de más de 15 días de evolución.

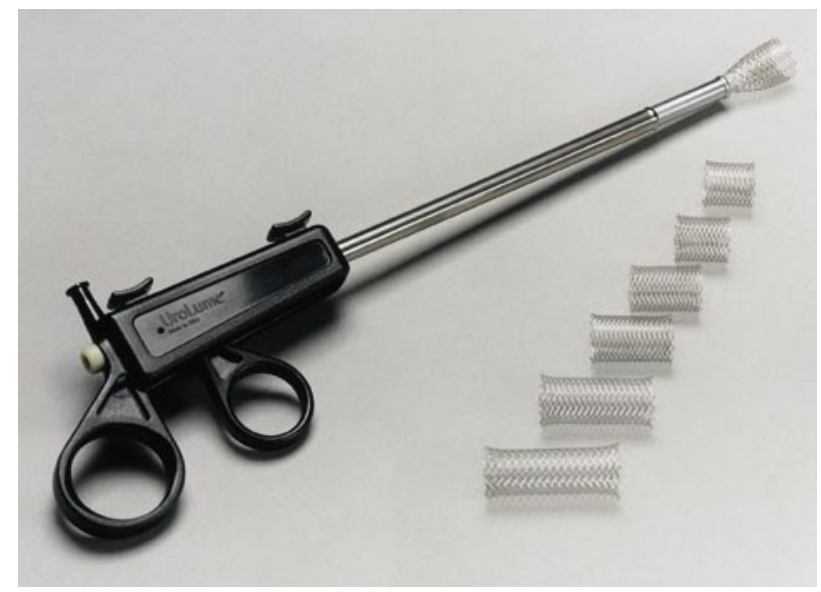

FIGURA 1. Prótesis de Urolume® (American Medical System) con aplicador de prótesis (24 Fr). 
2) Anestesia local de la uretra con gel de xilocaína durante 15 minutos (en pacientes con mucho riesgo quirúrgico, $\mathrm{ASA}$ IV) o locorregional en pacientes sin contraindicación quirúrgica.

3) Uretrotomía hasta un diámetro uretral mínimo $24 \mathrm{Fr}$ y medición de la estenosis uretral mediante el catéter uretral graduado de AMS o medición de la longitud de uretra prostática con el mismo catéter en el caso de obstrucción por HBP.

4) Implante de la prótesis bajo visión directa con el acoplador suministrado, siendo la longitud de la prótesis $1 \mathrm{~cm}$ más larga que la medición de la estenosis de uretra o $0.5 \mathrm{~cm}$ más corta que la medición de la longitud de urtetra prostática en los casos de HBP. El stent de UroLume ${ }^{\circledR}$ posee un calibre de $42 \mathrm{Fr}$ y la longitud de la prótesis varía entre $1.5 \mathrm{a} 4 \mathrm{~cm}$. El dispositivo se introduce con la ayuda de un acoplador de calibre $21 \mathrm{Fr}$ y un panendoscopio de 0 .

La valoración de los síntomas miccionales irritativos se realizó mediante la escala de MadesnIversen (10 síntomas urinarios con puntuación máxima de 3 en cada uno de ellos: tenesmo, chorro flojo, vaciado incompleto, frecuencia, nicturia, disuria, hematuria, micción en dos tiempos, goteo postmiccional, tiempo de micción prolongado). Esta misma escala se utilizó junto a la flujometría para evaluar la respuesta/mejoría tras la implantación de la endoprótesis.
El seguimiento de los pacientes lo relizamos además con Rx simple, cultivo de orina, ecografía a los 6 y 12 meses y ecografía transrectal de forma opcional.

\section{RESULTADOS}

La edad media de los pacientes con HBP fue de 72 años [(62-79), mediana de 75].

De los pacientes con HBP $(58.8 \%, n=10)$, el $80 \%(n=8)$ eran portadores de sonda.

El riesgo quirúrgico (ASA) de los pacientes con HBP fue de ASA III en el $40 \%$ y de ASA IV el $60 \%$.

La colocación de la endoprótesis se realizó con gel de xilocaína en 4 de estos 10 pacientes $(40 \%)$.

En la actualidad han fallecido 4 pacientes, llevando sólo 1 sonda permanente en el momento del éxitus. La técnica fracasó en 2 pacientes, uno requiriendo de sonda permanente por retención urinaria (hasta que falleció), y otro de extracción de la misma y adenomectomía por RAO.

Como complicaciones menores, la presencia de urgencia-frecuencia en 2 de los 6 pacientes vivos, uno de ellos con incontinencia leve.

\section{TABLA I. CRITERIOS DE EXCLUSIÓN.}

\begin{tabular}{|l|l|}
\hline ESTENOSIS RECURRENTES DE URETRA BULBAR & OBSTRUCCIÓN POR HBP \\
\hline Estenosis que no se pueda abrir hasta un 24 Fr & Obstrucción que no se pueda abrir/dilatar hasta un 24 Fr \\
\hline Estenosis que afecten al esfínter externo & Longitud de uretra prostática $>4 \mathrm{~cm}$ \\
\hline Infección urinaria activa & Infección urinaria activa \\
\hline Estrecheces supurativas & Tumor vesical \\
\hline Presencia de fístula en el área de implantación & Cáncer de próstata \\
\hline Tumor de células escamosas de uretra & Tumor de células escamosas de uretra \\
\hline Uretrostomía perineal & Litiasis vesical \\
\hline & Lóbulo medio voluminoso \\
\hline & Obstrucción de cuello vesical \\
\hline
\end{tabular}


La edad media de los pacientes con estenosis bulbar es de 44 años, significativamente menor a la media de edad de los pacientes con HBP [(30-79), mediana de 38], $p<0,005$.

En un caso tuvimos que colocar una segunda prótesis telescopada sobre la primera (por desplazamiento caudal de ésta), habiendo realizado además 2 RTU de tejido hiperplásico intraprotésico.

El seguimiento medio de los 17 pacientes ha sido de 41.2 meses (5.4-80.8).

De forma global y subjetivamente, la puntuación media en la escala de Madsen-lversen precirugía era de 22.5 frente a 7.78 postcirugía $(p<0,005$; test de Wilconson). También hubo mejorías objetivas en las medias de los flujos máximos pre y postcirugía (5.7 ml/s a 20.9; $p<0,005$; test de Wilconson).

\section{DISCUSIÓN}

La idea de utilizar un tutor para separar los lóbulos prostáticos parte de la utilización de stents en el sistema cardiovascular. Fabian (2) describió en 1980 por primera vez la utilización de tutores en urología y sugirió la aplicación de esta técnica en el tratamiento de la obstrucción del tracto de salida vesical secundario a HBP. Después de transcurridos algunos

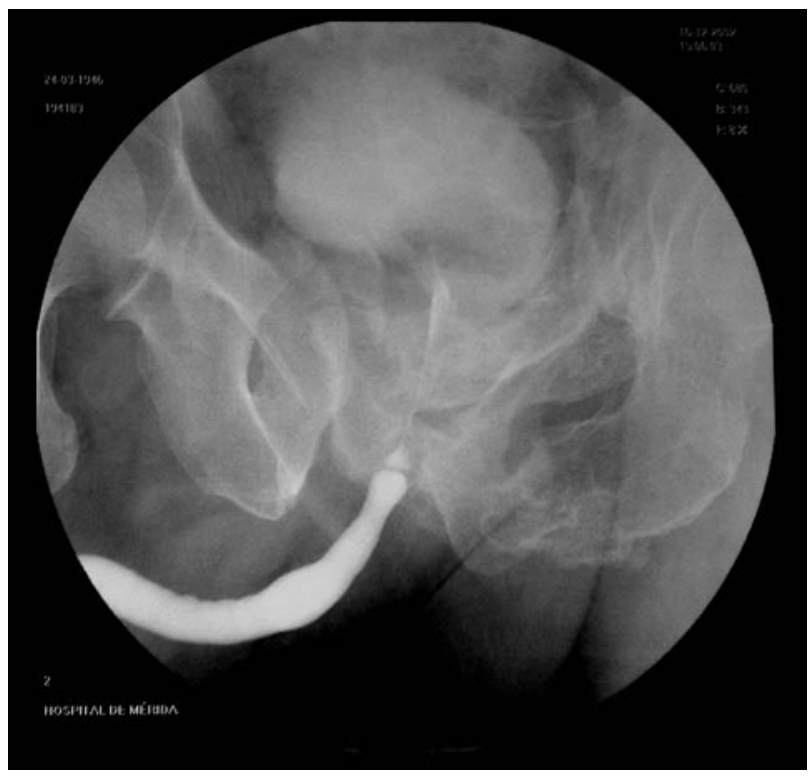

FIGURA 2. Uretrografía retrógrada en paciente con estenosis de uretra bulbar posterior de aproximadamente $1 \mathrm{~cm}$. Se observan signos de callos de fractura antiguos sobre tuberosidad isquiática izquierda. años se propuso el uso de tutores para el tratamiento de estenosis uretrales (3); a partir de entonces se generalizó su utilización intraprostática $(4,5)$. En este sentido, E. Milroy y R. Chapple publican en 1993 los resultados de 54 pacientes a los que se les colocó la prótesis de UroLume $\AA$ por HBP estando el 74\% (40 pacientes) totalmente satisfechos o con mínimos efectos secundarios al año de seguimiento.

En un ensayo multicéntrico abierto de mayor envergadura, Oesterling y cols. (6) comunicaron los resultados del tratamiento de 126 hombres que se presentaron con síntomas del tracto urinario inferior moderados o severos (95 casos) o retención urinaria (31 casos). En el grupo de pacientes sin retención urinaria 80 de los 95 sujetos se consideraron evaluables a los 12 meses y 52 a los 24 meses; la puntuación en la escala de síntomas de Madsen disminuyó de 14 a 5.9 y 5.4 respectivamente. En el grupo con retención urinaria, los 24 pacientes (de 31 ) evaluables a los 12 meses presentaron una puntuación media de 6.1. En el grupo sin retención urinaria, la velocidad máxima del flujo urinario aumentó de 9.1 a 13 y 13.1 respectivamente. En un $10 \%$ de los casos se observaron síntomas de irritación durante la micción.

En otro estudio, Amon Sesmero y cols. (7) presentan los resultados de 78 varones con obstrucción infravesical por HBP, estando 73 de ellos con sonda permanente y siendo el $88 \%$ ASA IV. Al año del im-

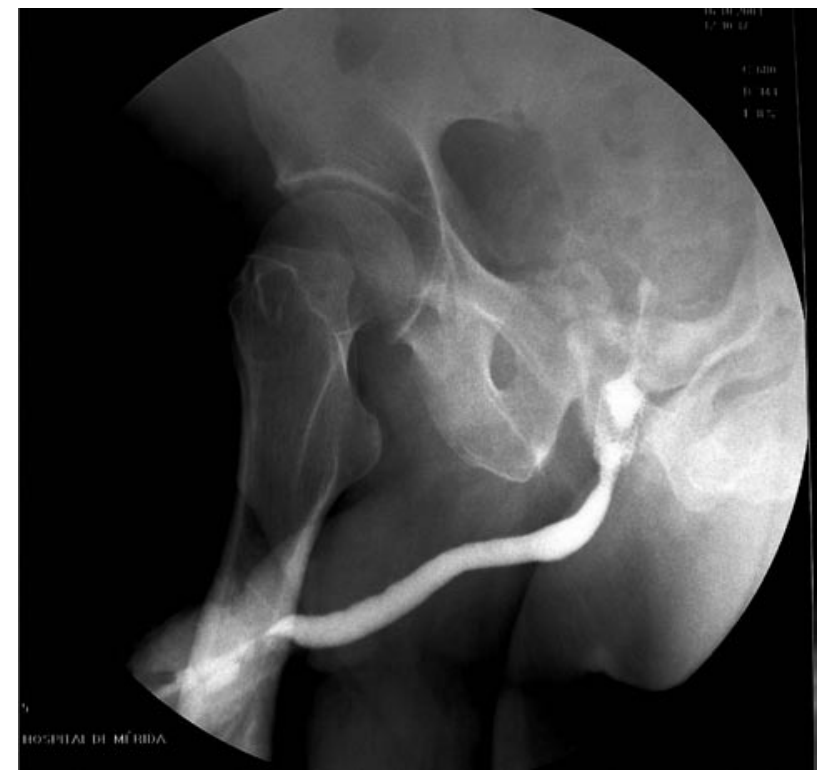

FIGURA 3. Crecimiento de tejido hiperplásico intraprotésico que ocluye la luz de la prótesis. 
plante las medias del flujo máximo y de la escala de síntomas I-PSS fueron de $12.7 \mathrm{ml} / \mathrm{s}$ y de 6.2 puntos respectivamente. Como complicaciones refieren tres casos de hematuria varios meses después del implante (que se manejaron de forma conservadora), un paciente al que se le tuvo que hacer RTU de tejido hiperplásico intraprotésico y tres casos de retirada de la prótesis. El seguimiento medio fue de 15.3 meses.

En cuanto a las prótesis para el tratamiento de las estenosis de uretra recidivantes, Shah K. y cols. (8) presentan los resultados tras 11 años de seguimiento de 24 pacientes de un total de 179 a los que se les habían implantado la prótesis de UroLume ${ }^{\circledR}$. Tras este período, seguían habiendo diferencias estadísticamente significativas pre y postcirugía de flujos miccionales máximos $(9.5 \mathrm{ml} / \mathrm{s}$ y $20.9 \mathrm{ml} / \mathrm{s}$ respectivamente) y la puntuación en la escala de síntomas urinarios (11.3 y 3.04). Las mejorías fueron evidentes al año del implante y permanecieron estables durante el seguimiento. La epitelización de la prótesis fue completa (90\% de epitelización o más) ya al año del implante en más del $90 \%$ de pacientes. Se obtuvieron biopsias en aquellos pacientes con crecimiento de tejido intraprotésico siendo negativas.

Los tutores intrauretrales están indicados en los casos de estenosis de uretra bulbar debido a que en esta zona existe un mayor tejido esponjoso siendo menos susceptible esta área de erosión.

Los extremos de la prótesis deben sobrepasar y cubrir como mínimo $5 \mathrm{~mm}$ más allá del área estenótica por cada lado. El número máximo de tutores a colocar no está bien definido; en cambio, la colocación de otros tutores, excepto cuando se colocaron originariamente dos tutores que luego se desplazan separándose, es inútil (9).

El antecedente de una uretroplastia previa es una contraindicación relativa (8). También debe evitarse su colocación en uretras previamente radiadas ya que la posibilidad de epitelización de la prótesis es menor. Otro subgrupo de pacientes que tampoco son buenos candidatos para el UroLume ${ }^{\circledR}$ son aquellos con estrecheces asociadas con espongiofibrosis profunda, es decir, los que han sufrido lesiones con separación uretral y lesiones en silla de montar con fibrosis profunda.

Aunque la incidencia de crecimiento de tejido hiperplásico intraprotésico (CTH) es relativamente alta, su traducción clínica significativa es baja. La mayoría de investigadores piensan que esto ocurre con más frecuencia en las estenosis postraumáticas $(10,11)$ tal y como ocurrió en nuestro caso $n^{\circ} 13$. Se trataba de un varón de 57 años que sufrió un acciden- te de tráfico en 1988 con estenosis bulbar secundaria de $1 \mathrm{~cm}$ (Figura 2). Se le habían realizado 5 uretrotomías fuera de nuestro centro con anterioridad. Se le planteó la posibilidad de uretroplastia, pero la rechazó. Se optó por la colocación de prótesis de Urolume $₫$ entre las alternativas terapeúticas ofertadas, colocándose ésta en abril/2003. En octubre/2003 tuvimos que colocar una segunda prótesis telescopada sobre la primera por desplazamiento craneal de ésta. Ha precisado de 2 RTUs por obstrucción de la prótesis debido a crecimiento de tejido hiperplásico intraprotésico, estando tras 14 meses de seguimiento desde la última RTU (noviembre/2005) con un $Q_{\max }$ de $27 \mathrm{ml} / \mathrm{s}$ y $Q_{\text {med }}$ de $11.5 \mathrm{ml} / \mathrm{s}$ (Figura 3).

Si el tutor no funciona, probablemente sería prudente extraerlo para reconstruir la uretra, que es posible con resultados aceptables $186 \%$ con un seguimiento medio de 25 meses) (12).

Shah K. y cols. (13) revisan a 69 a los que se les extrajo la prótesis de Urolume $₫$ de un total de 465 implantes del The North American Study Group (14.8\% de la serie). El porcentaje de extracción fue del $23 \%$ en los pacientes con HBP, del $5 \%$ en pacientes con estenosis de uretra y del $22 \%$ en pacientes con disinergia vésico-esfinteriana. El $43.8 \%$ de los explantes se realizaron en el primer año. Las causas de exéresis fueron por movilización y anclaje incorrecto en el $38.4 \%$ de los casos.

Los stents implantados por HBP o disinergia vesico-esfinteriana se pueden extraer generalmente de forma endoscópica y en 1 sola pieza. Los stents bulbares requieren de una resección del tejido de recubrimiento con el asa de resección a baja potencia para después tirar de los alambres de la prótesis con pinzas de agarre y de esta forma proceder a su explante. En otras ocasiones la pieza no se puede extraer de forma completa y ha de ser removida pieza por pieza.

Otra opción es el uso de láser Holmium a baja potencia para la resección del tejido que recubre la superficie de la prótesis pudiendo extraerse a continuación con pinzas de agarre (14).

Elliot D. y cols. (15) describen el uso combinado de la prótesis de UroLume ${ }^{\circledR}$ y el esfínter urinario artificial para el manejo de la estenosis recidivante de cuello y la incontinencia urinaria post-prostatectomía radical en 9 pacientes consiguiendo una reducción en el número de empapadores de 6.5 a 0.7 tras un seguimiento medio de 17.5 meses.

En los casos de disinergia vésico-esfinteriana de pacientes con trauma espinal, se ha utilizado con 
resultados similares a la esfinterotomía ( $\mathrm{n}=57$ pacientes) (16).

También se ha descrito el uso de la prótesis de UroLume ${ }^{\circledR}$ en pacientes con retención urinaria aguda tras la braquiterapia, debiéndose retirar en 2 de 5 pacientes por disuria y dolor irradiado al pene (17).

\section{CONCLUSIONES}

El implante de la prótesis de Urolume ${ }^{T M}$ es una técnica segura y sencilla, pudiendo colocarse con anestesia local de forma ambulante. Presenta baja tasa de complicaciones, con mejoría significativa de los parámetros flujométricos y del cuestionario de síntomas. Constituyen una muy buena opción a tener en cuenta en pacientes ancianos con hiperplasia benigna de próstata, retencionistas crónicos y con alto riesgo quirúrgico o que rechazan la cirugía.

Su uso en la disinergia vésicoesfinteriana es una alternativa a tener en cuenta frente a la esfinterotomía.

En los casos de estenosis de uretra recidivante pueden ser una alternativa válida a la cirugía en pacientes de mediana edad con estenosis bulbares cortas, en los que no se hayan realizado colgajos con piel y que no tengan espongiofibrosis profunda.

\section{BIBLIOGRAFIA y LECTURAS RECOMENDADAS ( ${ }^{*}$ lectura de interés $y^{* *}$ lectura fundamental)}

1. DOTTER, C.: "Transluminally placed coilspring endarterial tube grafos: long term patency in canine popliteal artery". Invest Radiol, 4: 329, 1969.

2. FABIAN, K.M.: "The intraprostatic "partial catheter" (urological spiral)". Urologe A, 19: 236, 1980.

3. BADLANI, G.; PRESS, S.M.; DEFALCO, A. y cols.: "Urolume endourethral prosthesis for the treatment of urethral stricture disease: long-term results of the North American Multicenter". UroLume Trial Urology, 45: 846, 1995.

*4. MILROY, E.; CHAPPLE, C.R.: "The UroLume stent in the management of benign prostatic hyperplasia". J. Urol., 150: 1630, 1993.

5. VICENTE, J.; SALVADOR, J.; CHECHILE, G.: "Spiral urethral prosthesis as an alternative to sur- gery in high risk patients with benign prostatic hyperplasia: prospective study". J. Urol., 142: 1504, 1989.

**6. OESTERLING, J.E.; KAPLAN, S.A.; EPSTEIN, H.B. y cols.: "The North American experience with the UroLume endoprosthesis as a treatment for benign prostatic hyperplasia: long-term results. The North American UroLume Study Group". Urology, 44: 353, 1994.

*7. AMON SESMERO, J.H.; MARTÍNEZ-SAGARRA OCEJA, J.M.; ESTEBAÑEZ ZARRANZ J. y cols.: "Permanent endoprostatic prostheses in patient with obstruction caused by benign hyperplasia of the prostate". Actas Urol. Esp., 22: 490, 1998.

**8. SHAH, D.K.; PAUL, E.M.; BADLANI, G.H: "North American Study Group. 11-year outcome analysis of endourethral prosthesis for the treatment of recurrent bulbar urethral stricture". J. Urol., 170: 1255, 2003.

9. WALSH, P.; RETIK, A.; VAUGHAN. y cols.: Campbell Urología; $8^{\text {a }}$ Ed. Tomo IV; pags 42894291.

10. SNELLER, Z.; BOSH, R.: "Restenosis of the urethra despite indwelling wallstent". J. Urol., 148: 145, 1992.

11. BREAME, H.; VAN POPPEL, H.; VAN DER VOORDE, W. y cols: "Total fibrotic obliteration of urethral stent". Br. J. Urol., 72: 389, 1993.

12. BARBAGLI, G.; PALMINTERI, E.; GUASÓN, G.: "Surgical renoval of urethral stents". J. Urol., 161: 102, 1999.

**13. SHAH, D.K.; KAPOOR, R.; BADLANI, G.H.: "North American Study Group. Experience with urethral stent explantation". J. Urol., 169: 1398, 2003.

*14. COSTAS, D.L.; RAVI MUNVER, M.; PREMINGER.: "Renoval of a UroLume prostatic stent using the Holmium Laser". Urology, 57: 166, 2001.

15. ELLIOTT, D.S.; BOONE, T.B.: "Combined stent and artificial urinary sphincter for management of severe recurrent bladder neck contracture and stress incontinente alter prostatectomy: a longterm evaluiation". J. Urol., 165: 413, 2001.

16. CHANCELLOR, M.B.; BENNETT, C.; SIMONEAU, A.R. y cols.: "Combined stent and artificial urinary sphincter for management of severe recurrent bladder neck contracture and stress incontinente alter prostatectomy: a long-term evaluiation". J. Urol., 165: 413, 2001.

17. KONETY, B.R.; PHELAN, M.W.; O'DONNELL, W.F. y cols.: "UroLume stent placement for the treatment of postbrachytherapy bladder outlet obstruction". Urolgy, 55: 721, 2000. 